\title{
CLASSIFICAÇÃO MACROSCÓPICA, IDENTIFICAÇÃO DA MICROBIOTA FÚNGICA E PRODUÇÃO DE AFLATOXINAS EM HÍBRIDOS DE MILHO
}

\author{
MACROSCOPIC CLASSIFICATION, IDENTIFICATION OF FUNGAL MICROBIOTA \\ AND AFLATOXINS PRODUCTION IN CORN HYBRIDS
}

\author{
Paulo Dilkin ${ }^{1}$ Carlos Augusto Mallmann ${ }^{2}$ Janio Morais Santurio $^{3}$ \\ José Luis Hickmann ${ }^{4}$
}

\section{RESUMO}

Com o objetivo de medir o potencial de resistência de 5 diferentes híbridos de milho, logo após a colheita, ao cres cimento de fungos e produção de aflatoxinas (AFs), foram avaliados os seguintes parâmetros: 1) aspecto macroscópico dos grãos, sendo os grãos de cada híbrido classificados como íntegros, danificados por insetos (DI) ou danificados por fungos $(D F)$; 2) contaminação fúngica dos híbridos; 3) potencial para resistência à produção de AFs, através do cultivo de Aspergillus parasiticus, linhagem NRRL 2999, sobre grãos de cada híbrido estudado; 4) consumo de matéria seca dos híbridos pelo cultivo fúngico. Como resultado, observou-se que $38 \%$ do milho de todos os híbridos apresentaram comprometimento macroscópico, sendo $26,7 \%$ DI e 11,3\% DF. Os híbridos recém-colhidos apresentaram contaminação fúngica por Penicillium sp. (14,3\%); Aspergillus sp. $(23,6 \%)$ e Fusarium sp. $(57,1 \%)$. O potencial de produzir AFs pelos diferentes híbridos em cultivos por 5 e 10 dias apresentou diferença somente com relação à aflatoxina $G_{2}$ em cultivos por 5 dias. A média de consumo de matéria seca dos híbridos de milho foi de 1,25 e 2,69\% submetidos ao cultivo de fungos por períodos de 5 e 10 dias, respectivamente.

Palavras-chave: milho, micotoxinas, aflatoxinas

\section{SUMMARY}

To determine the resistance of five different recently harvested corn hybrids to fungal growth and aflatoxins (AFs) production the following parameters were measured: 1) Macroscopic aspect of the grain and each hybrid classified as whole, damaged by insects (DI) or damaged by fungi (DF); 2) Fungal contamination of hybrids 3) Resistance to AFs production, through the culture of Aspergillus parasiticus, NRRL 2999 strain, on each hybrid grain studied and 4) Consumption of dry matter of hybrids by the fungal culture. It was observad that $38 \%$ of all corn hybrids had macroscopic damage, being $26.7 \%$ DI and $11.3 \% \mathrm{DF}$. The recently harvested hybrids showed fungal contamination by Penicillium sp. (14.3\%); Aspergillus sp. (23.6\%) and Fusarium sp. (57.1\%). The production of AFs by the different hybrids cultured for 5 and 10 days showed difference only when compared to aflatoxin $G_{2}$ cultured for 5 days. The average dry matter consumption of corn hybrids was of 1.25 and $2.69 \%$, submitted to fungal culture for 5 and 10 days respectively.

Key worn: corn, mycotoxins, aflatoxins

\section{INTRODUÇÃO}

Uma grande quantidade de fungos toxigênicos podem contaminar os cereais, principalmente em regiões de clima tropical. ASEVÊDO $\boldsymbol{e t}$ al. (1994) e ORSI et al. (1995) demostraram que o gênero fúngico mais encontrado no milho recémcolhido foi Fusarium, seguido por Aspergillus e Penicillium. Levantamento feito por SALGADO $\boldsymbol{e t}$ al. (1980) em amostras de milho, trigo e arroz, provenientes do estado de Santa Catarina, mostrou que 90\% das amostras eram positivas para Fusarium moniliforme e Fusarium graminearum. Pozzi, apud CORRÊA (1995), pesquisou mensalmente a microbiota fúngica de 130 amostras de milho. Os gêneros mais encontrados foram: Fusarium 83,8\%; Penicillium 55,3\% e Aspergillus em 40,7\% das amostras analisadas.

\footnotetext{
Médico Veterinário, Mestre em Medicina Veterinária Preventiva.

${ }^{2}$ Médico Veterinário, Doutor, Professor Titular, Departamento de Medicina Veterinária Preventiva, Universidade Federal de Santa Maria (UFSM), 97105-900, Santa Maria, RS. E-mail: mallmann@ccr.ufsm.br. Autor para correspondência.

${ }^{3}$ Médico Veterinário, Especialista, Professor Adjunto, Departamento de Medicina Veterinária Preventiva, UFSM.

${ }^{4}$ Acadêmico do Curso de Medicina Veterinária, UFSM.
} 
A contaminação dos cereais por fungos toxigênicos e produção de micotoxinas nos mesmos podem dar-se ainda no período pré-colheita, dependendo da espécie de planta envolvida, fatores geográficos e climáticos e manipulação dos mesmos na colheita, transporte, secagem, armazenamento e beneficiamento (ANDERSON et al., 1975; FENNELL et al., 1975; LILLEHOJ, et al., 1976; ORGANISACIÓN MUNDIAL DE LA SALUD, 1983; CRUZ, 1995). ZERINGUE et al. (1996) sugerem que as variações nas concentrações de aflatoxinas (AFs), encontradas em diferentes híbridos de milho, estejam relacionadas à concentração de ácido linoleico e da enzima lipoxigenase. A lipoxigenação do ácido linoleico seria capaz de produzir aldeídos voláteis com cadeias de 6 até 12 carbonos, inibindo ou impedindo o crescimento de fungos e a formação de AFs.

As AFs são metabólitos secundários de fungos das espécies Aspergillus flavus e Aspergillus parasiticus. Segundo LEESON et al. (1995), cerca de $50 \%$ das espécies de $\boldsymbol{A}$. flavus e $\boldsymbol{A}$. parasiticus são produtores de AFs. Somente quatro foram identificadas como contaminantes naturais de produtos agrícolas. São denominadas aflatoxina $\mathrm{B}_{1},\left(\mathrm{AFB}_{1}\right)$; $\mathrm{B}_{2},\left(\mathrm{AFB}_{2}\right) ; \mathrm{G}_{1},\left(\mathrm{AFG}_{1}\right) ; \mathrm{G}_{2},\left(\mathrm{AFG}_{2}\right)$. A $\mathrm{AFB}_{1}$ é a mais tóxica do grupo, seguida pela $\mathrm{AFG}_{1}, \mathrm{AFB}_{2} \mathrm{e}$ $\mathrm{AFG}_{2}$ com toxicidade de $50 \%, 20 \%$ e $10 \%$ em relação à primeira, respectivamente (SHOTWELL $\boldsymbol{e t}$ al., 1966; LEESON et al., 1995).

Em cereais estocados, os fatores mais importantes para o crescimento de fungos toxigênicos do gênero Aspergillus e a produção de AFs são a umidade relativa do ar e do substrato e temperatura de armazenamento. Umidade relativa de 80 a $85 \%$ com $17 \%$ de umidade dos cereais e temperatura de 24 a $35^{\circ} \mathrm{C}$ são condições ótimas para a produção de AFs (LEESON et al., 1995). O crescimento fúngico dificilmente ocorre em cereais que apresentam umidade inferior a 12\% (REDDY, 1992). Em rações, ótimas condições para a produção de AFs foram encontradas com umidade de 10 a $13 \%$ do substrato, 79 a $89 \%$ de umidade relativa do ar e temperaturas de 19 a $27^{\circ} \mathrm{C}$ (JONES et al., 1982). Segundo ORGANISACIÓN MUNDIAL DE LA SALUD (1983), as temperaturas mínima, ótima e máxima para a produção das $\mathrm{AFs}$ são de $12^{\circ} \mathrm{C}, 27^{\circ} \mathrm{C}$ e 40 $42^{\circ} \mathrm{C}$, respectivamente.

Este estudo objetivou a análise da contaminação natural por fungos e micotoxinas de cinco híbridos de milho. Quantificou-se a contaminação pelos gêneros Aspergillus, Penicillium e Fusarium. As micotoxinas pesquisadas foram: AFs, ocratoxina $\mathrm{A}$, zearalenona e fumonisina $\mathrm{B}_{1}$. Em paralelo, classificaram-se também os diferentes híbridos em percentagem de grãos íntegros, danificados por insetos e danificados por fungos, e cultivou-se Aspergillus parasiticus linhagem NRRL 2999 no milho íntegro para comparar o potencial de produção de AFs entre os diferentes híbridos e o consumo de matéria seca.

\section{MATERIAL E MÉTODOS}

Os cinco híbridos de milho avaliados, numerados de 1 a 5 , foram coletados aleatoriamente de uma lavoura experimental do Departamento de Solos do Curso de Agronomia da Universidade Federal de Santa Maria, onde se cultivaram outros 20 híbridos de diferentes marcas comerciais. O cultivo foi conduzido em parcelas de $60 \mathrm{~m}^{2}$ com três repetições para cada híbrido. De cada híbrido foram colhidos aproximadamente $20 \mathrm{~kg}$ de milho em espiga, que foi debulhado manualmente, evitando-se dano mecânico. Essa foi realizada no mês de março de 1996, após a maturação fisiológica do cereal.

\section{Quantificação da contaminação fúngica dos hí- bridos}

A quantificação da contaminação fúngica foi realizada conforme a metodologia preconizada por SAMSON et al. (1996). Colocaram-se 10 grãos de milho no meio de DRBC AGAR BASE ${ }^{\circledR}$ (OXOID UNIPATH Ltda., Basingstoke, Hampshire, England), em placa de Petri, com cinco repetições por híbrido. O período de incubação foi de 5 dias a $25^{\circ} \mathrm{C}$. Em seguida, fez-se a identificação dos fungos que se desenvolveram nos grãos com o auxílio de estereoscópio (Leitz Wetzlar, modelo 490348) e microscópio (Leitz Wetzlar, modelo 736916).

\section{Classificação macroscópica dos híbridos de milho}

Realizaram-se a seleção e separação manual de três amostras de $1 \mathrm{~kg}$ de cada híbrido em grãos íntegros, os que não apresentaram lesões provocadas por insetos ou alteração da coloração provocada pelo desenvolvimento fúngico (ardido). $\mathrm{O}$ segundo grupo foi formado por grãos danificados por insetos (DI) e outro por grãos que apresentaram danos provocados pelo desenvolvimento fúngico (DF). Grãos de todos os híbridos, que não apresentaram danos físicos nem contaminação fúngica aparente, foram selecionados para a inoculação fúngica e cultivo para a avaliação do potencial de produção de AFs de cada híbrido.

\section{Preparação dos híbridos de milho e inoculação fúngica}

As amostras foram submetidas ao exame micotoxicológico prévio, avaliando-se a contaminação por AFs, ocratoxina A, zearalenona e fumonisina $B_{1}$. As amostras negativas foram utilizadas para $o$ cultivo de fungo com o objetivo de avaliar o potencial de produção de AFs de cada híbrido. 
A metodologia empregada para esterilizar o milho e inoculação fúngica foi adaptada da técnica preconizada por PITT et al. (1992). A esterilização da superfície dos grãos de milho foi realizada através da imersão das amostras em solução de hipoclorito de sódio a $0,4 \%$ por 2 minutos e, após, lavagem em água estéril, empregando-se essa forma de esterilização para minimizar a interferência na composição do milho. A seguir, as amostras de 100 gramas de milho foram acondicionadas em frascos Erlernmeyer de $250 \mathrm{~m} \ell$, aumentando-se a umidade das amostras de milho para $18 \%$ através da adição de água deionizada. Os frascos foram fechados com tampas de algodão. A inoculação de esporos de Aspergillus parasiticus linhagem NRRL 2999 foi realizada através da adição de uma solução de TRITON X $100^{\circledR}$ (MERCK S. A. Indústrias Químicas, Estrada dos Bandeirantes, 1099. Rio de Janeiro, RJ) a $0,5 \%$ em água. A padronização do inóculo foi realizada após o quinto dia de incubação do fungo. Contou-se o número de unidades formadoras de colônias (UFC/m $\ell$ ), utilizando-se o hemocitômetro de Neubauer. A seguir, ajustou-se o inóculo para $5 \mathrm{x}$ $100.000 \mathrm{UFC} / \mathrm{m} \ell$, diluindo-se com o próprio meio de cultivo, de acordo com a metodologia descrita por SHADOMY et al. (1985). Inoculou-se aproximadamente 650.000 esporos para amostras de 100 gramas de milho.

O delineamento experimental foi de blocos ao acaso com três repetições para cada híbrido, nos períodos de 5 e 10 dias de incubação, totalizando 30 tratamentos. Os dados foram avaliados estatisticamente, empregando-se o teste Tukey $(\mathrm{p}<0,5)$.

\section{Produção e quantificação das aflatoxinas}

Os cultivos para a produção das AFs foram incubados em estufa BOD com temperatura constante de $25^{\circ} \mathrm{C}$ por períodos de 5 e 10 dias.

A extração das AFs produzidas e quantificação por Cromatografia de Camada Delgada foram realizadas conforme a metodologia preconizada por SOARES (1987). O limite de quantificação desse método foi de $1 \mu \mathrm{g} / \mathrm{kg}, 10 \mu \mathrm{g} / \mathrm{kg}$ e $50 \mu \mathrm{g} / \mathrm{kg}$ para AFs, ocratoxina A e zearalenona, respectivamente. Já para fumonisina $\mathrm{B}_{1}$, empregou-se a metodologia preconizada por BINKERD et al. (1993), com limite de quantificação de $10 \mu \mathrm{g} / \mathrm{kg}$.

\section{RESULTADOS E DISCUSSÃO}

Na tabela 1, pode-se observar que $38 \%$ do milho recém-colhido apresentou ação degenerativa, compreendendo os grãos DI $(26,7 \%)$ e DF $(11,3 \%)$. $\mathrm{O}$ efeito grãos DI foi estatisticamente superior aos
Tabela 1 - Classificação macroscópica de cinco híbridos de milho recém-colhidos. Porcentagem média de três repetições.

\begin{tabular}{cccc}
\hline Híbrido & Íntegro & $\begin{array}{c}\text { Danificados por } \\
\text { insetos }\end{array}$ & $\begin{array}{c}\text { Danificados por } \\
\text { fungos }\end{array}$ \\
\hline & & & \\
1 & $52,9^{\mathrm{a}}(10,8)^{*}$ & $31,8^{\mathrm{b}}(10,2)$ & $15,3^{\mathrm{c}}(2,5)$ \\
2 & $65,4^{\mathrm{a}}(10,1)$ & $27,1^{\mathrm{b}}(9,3)$ & $7,5^{\mathrm{c}}(2,9)$ \\
3 & $64,9^{\mathrm{a}}(3,2)$ & $21,0^{\mathrm{b}}(3,6)$ & $14,1^{\mathrm{c}}(1,5)$ \\
4 & $70,1^{\mathrm{a}}(21,1)$ & $22,5^{\mathrm{b}}(22,4)$ & $7,4^{\mathrm{c}}(1,6)$ \\
5 & $56,7^{\mathrm{a}}(15,0)$ & $31,1^{\mathrm{b}}(13,5)$ & $12,2^{\mathrm{c}}(3,8)$ \\
& & & 11,3 \\
Média & 62,0 & 26,7 & \\
& \\
* Desvio padrão. & \\
a-c Médias seguidas pela mesma letra na vertical não diferem entre \\
si (Tukey, p>0,05).
\end{tabular}

grãos DF em todos os híbridos, porém, dentro dos grupos não houve diferença significativa. Pode-se observar ainda que a porcentagem de milho DF é bastante alta, superior ao limite máximo tolerado pelas agroindústrias, que é de $6 \%$. Isso ocorreu provavelmente em função do milho ter permanecido na lavoura além do tempo ideal para colheita.

Apesar de encontrar alta incidência de fungos no milho recém-colhido (tabela 2) e umidade média de $18 \%$, não foram detectadas micotoxinas. Isso pode ser atribuído ao curto espaço de tempo para a formação das mesmas, ou número reduzido de linhagens fúngicas com potencial toxigênico. Não foi constatada diferença significativa com relação à incidência de fungos nos diferentes híbridos. Porém, a contaminação por Fusarium sp. nos híbridos de milho recém-colhidos foi significativamente superi-

Tabela 2 - Porcentagem de grãos com fungos potencialmente toxigênicos em cinco híbridos de milho recémcolhidos.

\begin{tabular}{|c|c|c|c|}
\hline Híbrido & Aspergillus sp. & Penicillium sp. & Fusarium sp. \\
\hline 1 & $16,0^{\mathrm{a}}(7,2)^{*}$ & $22,0^{\mathrm{a}}(22,7)$ & $65,3^{\mathrm{b}}(18,6)$ \\
\hline 2 & $12,7^{\mathrm{a}}(4,2)$ & $18,0^{\mathrm{a}}(5,3)$ & $58,2^{\mathrm{b}}(10,6)$ \\
\hline 3 & $39,3^{\mathrm{a}}(25,7)$ & $17,5^{\mathrm{a}}(21,9)$ & $59,3^{\mathrm{b}}(11,0)$ \\
\hline 4 & $28,0^{\mathrm{a}}(12,2)$ & $8,0^{\mathrm{a}}(2,0)$ & $48,0^{\mathrm{b}}(6,0)$ \\
\hline 5 & $22,0^{\mathrm{a}}(15,1)$ & $6,0^{\mathrm{a}}(6,9)$ & $54,7^{\mathrm{b}}(9,1)$ \\
\hline Média & $23,6^{\mathrm{a}}$ & $14,3^{\mathrm{a}}$ & $57,1^{\mathrm{b}}$ \\
\hline
\end{tabular}

Ciência Rural, v. 30, n. 1, 2000. 
or aos gêneros Aspergillus sp. e Penicillium sp. Na avaliação da microbiota fúngica, observouse ainda que nem todas as amostras apresentaram contaminação fúngica e outras apresentaram contaminação dupla ou até tripla.

A quantidade de fungos contaminantes encontrados no milho recém-colhido foi menor que a contaminação encontrada por ORSI et al (1995), porém a proporcionalidade dos três gêneros foi idêntica. Já ASEVÊDO et al. (1994), ao avaliarem a incidência de fungos em milho estocado, encontraram com maior freqüência o gênero Aspergillus (72,2\%), seguido pelo Penicillium $(67,7 \%)$ e Fusarium $(62,2 \%)$, diferindo das proporcionalidades normalmente encontradas no milho recém-colhido.

Nas tabelas 3 e 4, podem ser observados os resultados da produção de AFs após cultivo de Aspergillus parasiticus linhagem NRRL 2999, em cinco híbridos de milho. Esse fungo mostrou-se grande produtor de AFs no milho, especialmente $\mathrm{AFG}_{1}$. No entanto, SHOTWEL et al. (1966), cultivando a mesma linhagem em arroz, obtiveram alta produção de $\mathrm{AFB}_{1}$. Houve diferença significativa de AFs produzidas pelos diferentes híbridos somente quanto à produção de $\mathrm{AFG}_{2}$ em cultivos de 5 dias . De acordo com as constatações de ZERINGUE $\boldsymbol{e t}$ al. (1996), pode-se estimar que os híbridos analisados possuem níveis de ácido linoleico e produção de lipoxigenase similares, propiciando condições semelhantes aos Aspergillus sp. para a produção de AFs.

Tabela 3 - Média de aflatoxinas produzidas $(\mu \mathrm{g} / \mathrm{kg})$ em cinco híbridos de milho, em cultivos por 5 dias.

\begin{tabular}{cccccc}
\hline Híbrido & $\mathrm{AFB}_{1}$ & $\mathrm{AFB}_{2}$ & $\mathrm{AFG}_{1}$ & $\mathrm{AFG}_{2}$ & Total \\
& & & & & \\
\hline & & & & & \\
1 & $3152^{\mathrm{a}}(2102,8)^{*}$ & $66^{\mathrm{a}}(0,1)$ & $25955^{\mathrm{a}}(12588,1)$ & $527^{\mathrm{ab}}(1,8)$ & $29700^{\mathrm{a}}$ \\
2 & $6370^{\mathrm{a}}(1379,2)$ & $127^{\mathrm{a}}(104,9)$ & $19249^{\mathrm{a}}(16159,5)$ & $657^{\mathrm{b}}(71,2)$ & $26403^{\mathrm{a}}$ \\
3 & $4427^{\mathrm{a}}(3143,4)$ & $82^{\mathrm{a}}(1,6)$ & $3997^{\mathrm{a}}(4662,5)$ & $287^{\mathrm{a}}(71,2)$ & $8793^{\mathrm{a}}$ \\
4 & $3228^{\mathrm{a}}(2033,9)$ & $74^{\mathrm{a}}(9,1)$ & $13704^{\mathrm{a}}(20500,1)$ & $422^{\mathrm{ab}}(85,9)$ & $17428^{\mathrm{a}}$ \\
5 & $5362^{\mathrm{a}}(511,9)$ & $73^{\mathrm{a}}(1,1)$ & $10590^{\mathrm{a}}(7075,2)$ & $547^{\mathrm{ab}}(156,9)$ & $16572^{\mathrm{a}}$
\end{tabular}

*Desvio padrão.

${ }^{a-b}$ Médias seguidas pela mesma letra na vertical não diferem entre si (Tukey, p>0,05).
Constatou-se, também, que houve diferença significativa com relação ao consumo de matéria seca do milho somente nos diferentes períodos de cultivos fúngicos, com médias de 1,25 e $2,69 \%$ em cultivos de com 5 e 10 dias, respectivamente (tabela $5)$.

\section{CONCLUSÃO}

Dos híbridos avaliados, 62,0\% dos grãos apresentaram-se íntegros, $26,7 \%$ danificados por insetos e 11,3\% danificados pelo desenvolvimento fúngico. Não houve diferença significativa entre os cinco híbridos avaliados, tanto no grupo danificados por insetos ou danificados pelo desenvolvimento fúngico. Os híbridos estavam contaminados por Fusarium sp. 57,1\%; Aspergillus sp. 23,6\% e Penicillium sp. $14,3 \%$. Não houve diferença significativa entre os híbridos avaliados em relação à incidência dos três gêneros fúngicos, bem como esses não apresentaram níveis detectáveis de aflatoxinas, zearalenona, ocratoxina $\mathrm{A}$ e fumonisina $\mathrm{B}_{1}$. Os híbridos avaliados apresentaram diferença significativa quanto ao potencial de produzir aflatoxinas somente de aflatoxina $\mathrm{G}_{2}$ em cultivos de Aspergillus parasiticus por 5 dias. $\mathrm{O}$ crescimento fúngico no milho implicou a diminuição da matéria seca do milho, sendo de 1,25 e 2,69\% em cultivos de Aspergillus parasiticus por períodos de 5 e 10 dias, respectivamente. 
Tabela 5 - Consumo de matéria seca em cinco híbridos de milho incubados com Aspergillus parasiticus. Porcentagem média de três repetições.

\begin{tabular}{|c|c|c|}
\hline \multirow[t]{2}{*}{ Híbrido } & \multicolumn{2}{|c|}{ Dias de cultivo } \\
\hline & Cinco & Dez \\
\hline 1 & $1,21^{\mathrm{a}}(0,05)^{*}$ & $2,68^{\mathrm{b}}(0,06)$ \\
\hline 2 & $1,23^{\mathrm{a}}(0,05)$ & $2,71^{\mathrm{b}}(0,08)$ \\
\hline 3 & $1,18^{\mathrm{a}}(0,08)$ & $2,62^{\mathrm{b}}(0,18)$ \\
\hline 4 & $1,23^{\mathrm{a}}(0,04)$ & $2,68^{\mathrm{b}}(0,13)$ \\
\hline 5 & $1,25^{\mathrm{a}}(0,02)$ & $2,75^{\mathrm{b}}(0,04)$ \\
\hline Média & 1,22 & 2,69 \\
\hline
\end{tabular}

\section{REFERÊNCIAS BIBLIOGRÁFICAS}

ANDERSON, H.W., NEHRING, E. W., WICHSER, N. Aflatoxin contamination of corn in the field. Journal of Agricultura Food Chemistry, Washington, USA, v. 23, p. 775-782, 1975.

ASEVÊDO, I.G., GAMBALE, W., CORRÊA, B., $\boldsymbol{e}$ t $\boldsymbol{a}$ l. Mycoflora and aflatoxigenic species of Aspergillus spp isolated from stored maize. Revista de Microbiologia, São Paulo, v. 25, n. 1 p. 46-50, 1994.

BINKERD, K.A., SCOTT, D.H., EVERSON, R.J. et al. Fumonisin contamination of the 1991 Indiana corn crop and its effects on horses. Journal Veterinary Diagnostic Investigation, Lawrence, USA, v. 5 p. 653-655, 1993

CORRÊA, B. Fungos toxigênicos em grãos e rações: biologia, ocorrência e controle. In: SIMPÓSIO INTERNACIONAL SOBRE MICOTOXINAS E MICOTOXICOSES EM AVES. 1995, Curitiba, PR. Anais... Curitiba, FACTA: Fundação de Apoio à Ciência e Tecnologia Avícolas, 1995. 332 p. p. 15-20.

CRUZ, L.C.H., Características gerais das micotoxinas e micotoxicoses. In: SIMPÓSIO INTERNACIONAL SOBRE MICOTOXINAS E MICOTOXICOSES EM AVES, 1995, Curitiba, PR. Anais... Curitiba, FACTA: Fundação de Apoio à Ciência e Tecnologia Avícolas, 1995. 332 p. p. 1-13.

FENNELL, D.I., LILLEHOJ, E.B., KWOLEK, W.F. Aspergillus flavus and other fungi associated with insect damaged field corn. Cereal Chemistry. St. Paul, USA, v. 52, p. 314-321, 1975 .

JONES, F.T., HAGLER, W.H., HAMILTON, P.B. Association of low levels of aflatoxin in feed with productivity losses in comercial broiler operations. Poultry Science, Savoy, USA, v. 61, p. $861-868,1982$
LEESON, S., GONZALO, J.D.G., SUMMERS, J.D. Poultry disorders and mycotoxins. Ontário, Canadá: Guelph, 1995. $350 \mathrm{p}$.

LILLEHOJ, E.B., FENNELL, D.I., HESSELTINE, C.W. Aspergillus flavus infection and aflatoxin production in mixtures of high-moisture and dry maize. Journal of Stored Products Research, v. 12, p. 11-18, 1976.

ORSI, R.B., CORRÊA, B., POZZI, C.R., et al . Microbiota fúngica em três híbridos de milho recém colhidos e armazenados. In: III SEMINÁRIO SOBRE A CULTURA DO MILHO "SAFRINHA", Assis, SP. 1995. Anais... Assis: Instituto Agonômico de São Paulo, 1995. p. 105-110.

ORGANISACIÓN MUNDIAL DE LA SALUD. Critérios de salud ambiental 11: Micotoxinas. Cidade de México: OSP, 1983. $131 \mathrm{p}$

PITT, J.I., HOCKING, A.D., SAMSON, R.A. Recommended methods for mycological examination of foods. In: SAMSON, R. A., HOCKING, A. D., PITT, J. I. et al. Modern methods in food mycology. Amsterdam: Centraalbureau voor Schimmelcultures, 1992. Cap. 3, p. 365-368.

REDDY, C.V. Aflatoxins in feed: an enemy to poultry producers in the tropics. World Poultry, Doetichen, Netherland, v. 8, p. 19-20, 1992.

SALGADO, I.M., CARVALHO, P.C.T. Fungos toxigênicos associados a cereais. Levantamento da micoflora associada a milho, trigo e arroz. Revista de Microbiologia, São Paulo, v. 11 , p. $60-63,1980$.

SAMSON, R.A., HOEKSTRA, E.S., FRISVAD, J.C. Introduction to food-Borne fungi. 5. ed. Netherlands: Centraalbureau voor Schimmelcultures, 1996. 322 p.

SHADOMY, S., ESPINEL-INGROFF, A., CARTWRIGHT, R. Laboratory studies with antifungal agents: susceptibility tests and biossays. In: LENNETE, E.H., BALLOWS, A., HAVSLER, Jr. W.J., et al. Ed. Manual of clinical Microbiology, 4. ed. Washington: American Societty of Microbiology, 1995. Cap. 5, p. 991-999.

SHOTWELL, O.L., HESSELTINE, C.W., STUBLEFIELD, R.D., et al. Production of aflatoxin on rice. Applied Microbiology, Michigan, USA, v. 14, n. 3, p. 425-428, 1966.

SOARES, L.M.V. Micotoxinas: Um método para análise simultânea e incidência em alimentos comercializados na região de Campinas. Campinas, São Paulo: Unicamp, 1997. 129p. Tese (Doutorado em Engenharia de Alimentos) - Faculdade de Engenharia de Alimentos e Agrícola, Universidade Estadual de Campinas, 1987.

ZERINGUE, H.J., BROWN, R.L., NEUCERE, J.N., $\boldsymbol{e} \boldsymbol{t} \boldsymbol{a l}$. Relationships between $\mathrm{C}_{6}-\mathrm{C}_{12}$ alkanal and alkenal volatile contents and resistance of maize genotypes to Aspergillus flavus and aflatoxin prodution. Journal of Agricultural Food Chemistry, Washington, USA, v. 44, p. 403-407, 1996. 